\title{
Innovation Opportunities in Critical Results Communication: Practical Solutions
}

\author{
Bruce I. Reiner
}

Published online: 14 August 2013

(C) Society for Imaging Informatics in Medicine 2013

\section{The Critical Results Communication Schema}

In conventional practice, the process of critical results communication (CRC) largely consists of a two-step process, where one party (i.e., CRC sender) conveys critical results data to a second party (i.e., CRC recipient), who subsequently initiates a responsive clinical action. This communication largely takes place through either verbal or written communication and involves non-standardized textual data. The communication responsibilities are frequently outsourced to nonphysician third parties, who have the potential to introduce error and misunderstanding to the CRC [1].

A preferred CRC process should consist of a series of predictable and sequential steps aimed at ensuring that the communication and understanding of the critical result is accurate, complete, understood in its entirety, acted upon in a timely fashion, and compliant with institutional, community, and societal standards for best clinical practice. The sequential steps in this optimized CRC schema are listed in Table 1 and begin with identification and classification of the finding/disease which fulfills criteria for a "critical result." The identification of critical results can be manual or automated in accordance with established practice guidelines and available technologies. In manual critical results identification, the radiologist interpreting the imaging dataset would identify a finding or disease of sufficient criticality based upon established or personal standards. In automated critical results identification, a supporting technology such as natural language processing or a form of artificial intelligence (e.g., neural network) would identify a finding or indication in the radiology report or order entry data to automatically trigger a CRC.

\section{B. I. Reiner $(\bowtie)$}

Department of Radiology, Veterans Affairs Maryland Healthcare System, 10 North Greene Street, Baltimore, MD 21201, USA

e-mail: breiner1@comcast.net
Once the CRC application has been activated, the next step in the CRC schema is creation of the critical results communication data. While conventional practice typically utilizes unstructured (i.e., non-standardized) data for CRC, a preferred method would be utilization of standardized data, which could in turn allow for the creation of a referenceable CRC database. Incorporation of imaging data directly into the CRC is arguably a more efficient and understandable means of communicating critical result findings in radiology practice [2], as opposed to use of text-based communication alone. At the same time, the manner in which data is presented can be customized in accordance with the specific preferences of the CRC recipient [3] with the goal of improving end user acceptance and understanding. This customized data presentation can be automatically performed and tied to each individual end user's authentication and identification.

In addition to data presentation, customization of the CRC can also be applied to the transmission process, where individual recipient end users can have preferred methods of how data is transmitted (which can in part be modified in accordance with the urgency of the CRC). Options for data transmission can include (but not be limited to) telephone, e-mail, instant messaging, facsimile, texting, or face to face interaction. The single most important factor is that the CRC is transmitted and received in a timely fashion and commensurate with the urgency and criticality of the critical result. When communication is performed electronically, time stamps can be automatically recorded which document the day/time of CRC transmission, the criticality and urgency of the critical result, the methods used in data transmission, and the identities of the sender and intended recipients.

Data is also automatically recorded during the next step of the CRC schema which consists of receipt and acknowledgment of the CRC. This step is fundamental to documenting that the critical results data has in fact been received by the intended recipient and appropriate clinical actions can be performed to address the critical result. An important component of this step is 
Table 1 Steps in the critical results communication (CRC) schema

1. Identification and classification of finding/disease criticality

2. Creation of critical results communication instrument

3. Transmission of critical results communication

4. Receipt and acknowledgment of critical results communication

5. Recipient feedback of understanding with option for additional consultation

6. Initiation of clinical intervention and follow-up actions

7. Diagnostic confirmation

8. Analysis of critical results data and compliance with established standards

the ability to unequivocally document the identity of the recipient of the CRC, which is often lacking and can be a subject of debate in conventional practice. By directly integrating authentication/ identification technologies such as biometrics into the $\mathrm{CRC}$ schema and technology in use, one can determine and document the identity of the recipient without any potential for future denial. In the event that an intended recipient does not acknowledge receipt of the $\mathrm{CRC}$ in a timely fashion, an escalation communication pathway can be automatically activated, which follows pre-defined rules in accordance with the urgency of the $\mathrm{CRC}$, institutional guidelines, and societal (i.e., best practice) standards $[4,5]$. By electronically documenting and time stamping all communication actions, a reliable and unimpeachable record is available for review and analysis with the goal of continuously improving operational efficiency and clinical outcomes.

While receipt acknowledgment is a fundamental requirement of $\mathrm{CRC}$, it does not conclusively ensure that the data communicated was completely understood on the part of the recipient and this misunderstanding (or incomplete understanding) can potentially serve as a source of medical error $[6,7]$. While conventional CRC efforts have attempted to remedy this potential source of error by having the recipient "read back" the message conveyed, this verbal reiteration does not always ensure cognitive understanding. One solution to more effectively address this potential disparity between acknowledgment and understanding is the inclusion of standardized data in the CRC which incorporates clinical and time urgency of the critical results, follow-up recommendations, the recipient's clinical feedback, and option for additional consultation. All of these data can be standardized and incorporated into the $\mathrm{CRC}$ database, thereby providing a valuable tool for longitudinal analysis.

Once the CRC data has been successfully transmitted, received, and understood, the next step is the initiation of clinical action. In conventional practice, the relative lack of data integration between the reporting system, picture archival, communication system (PACS), and electronic medical record (EMR) preclude the recipient physician from performing these clinical actions inside the same application as used in the CRC process. If for example, a radiologist identifies a questionable pulmonary embolus in the lung base of an abdominal CT exam, he/she may recommend a follow-up chest CT angiography exam for definitive diagnosis. If the $\mathrm{CRC}$ incorporated the annotated "key images" from the abdominal CT exam along with corresponding text data, the recipient physician would be able to directly visualize the critical result of concern, evaluate the radiologistgenerated diagnosis and recommendations, and independently determine the best course of clinical action. If the recipient physician agreed that the recommendation for chest CT angiography was warranted, he/she would have to log out of the CRC application and log into a separate radiology ordering application in order to place the emergent order for chest CT angiography. The ordering physician may or may not receive an exact scheduling time upon ordering and would in all likelihood have to wait until either notified by the interpreting radiologist (or a designated subordinate) or receiving the final report. Since this conventional workflow introduces additional time delays, workflow inefficiencies, and potential for error, a preferred solution would be to integrate these follow-up actions directly into the CRC schema and technology in use. The proposed innovation would perform this function by creating a direct link between the CRC follow-up recommendations and resulting physician orders. In this example, the $\mathrm{CRC}$ recipient would have the ability to "agree with" the recommendation for chest CT angiography and "order now." Since the order was generated in accordance with an initial $\mathrm{CRC}$, the system labels this newly ordered imaging study as a "critical results" exam, which automatically triggers a new CRC (i.e., one example of how an automated CRC is generated from order entry data). When the technologist and radiologist retrieve the order entry data, they are presented with the initial CRC data which includes the annotated key images and associated text data, which assists in protocol selection, interpretation, and creation of a new CRC. In the course of creating the new CRC, the previous CRC (from the abdominal CT exam) is automatically "linked" with the current CRC data (from the chest CT angiography exam). In this manner, the data from both $\mathrm{CRCs}$ are linked to one another and would be reviewable in tandem whenever someone was to open up either of these critical result communications. Clinicians would also be provided with the ability to "link" supporting clinical data to the radiology CRC. If for example, this same patient had a prior history of deep venous thrombosis which was documented on a previous discharge summary, the clinician could link this data in the CRC using the clinical feedback feature and either "insert" the entire document or "cut and paste" specific data from this document into the current CRC. This provides a mechanism for "linking" imaging and clinical data onto a single CRC. (In addition to manual data extraction and linking, this function could also be automatically performed through computerized data mining techniques using artificial intelligence in combination with natural language processing.) This example illustrates three important features of the 
proposed innovation, the ability to integrate clinical orders (tied to follow-up recommendations of the $\mathrm{CRC}$ ) into the $\mathrm{CRC}$ application, ability to link disparate data, and the ability to automatically generate a CRC from order entry data.

The next step in the CRC process is data confirmation, which provides a mechanism to document the diagnostic accuracy of the CRC being reported by the radiologist. This provides critical peer review data from the referring clinician, who often has access to relevant clinical and/or historical data not known by the radiologist at the time of image interpretation. In addition to providing feedback on the diagnostic accuracy of reported CRCs, this would also include feedback on cases in which critical results went unreported. This could consist of either a "missed" critical result diagnosis or a critical result which was documented in the radiology report, but was not communicated in accordance with established CRC criteria.

Data analysis serves as the final step in the CRC schema and can be applied to data captured at all individual steps in the CRC schema, as well as the collective CRC process. Since the proposed innovation is predicated upon the use of standardized data (which can consist of imaging, textual, numerical, graphical, and pictorial data), the creation of a referenceable database becomes a reality. The resulting analytics can be used for a myriad of functions including (but not limited to) research, education and training, decision support, creation of best practice (i.e., evidence-based medicine) guidelines, quality assurance, individual and institutional operational performance assessment, and clinical outcomes analysis. Derived analytics can be customized to the specific needs and preferences of individual and institutional healthcare providers with creation of automated alerts and prompts when pre-defined data thresholds are reached. Suppose for example, a particular clinician was demonstrated to have relatively poorer CRC response times than his/her peers within a given institution. In the course of analyzing technology use, it was learned that the physician in question was not taking full advantage of one of the notification features frequently used by his/her colleagues, which provides automated CRC alerts at 25,50 , and $75 \%$ time intervals of a pending CRC. By learning of this underutilized feature, the clinician could begin to take advantage of available technology, while also being presented with "before and after" analysis, which provides insight as to how CRC performance data changes through a specific intervention. This illustrates that the goal of these CRC analytics is not intended to be punitive, but instead to be educational and empowering, with the goal of improved clinical outcomes based upon objective and customizable data analysis.

\section{Creating the Technology}

In formulating a technology development plan, we will begin with the first step in the CRC schema, which is the identification of criticality. The technology created must support both manual and automated modes of operation. Manual mode is straightforward; the end user (e.g., radiologist) determines a threshold for criticality has been reached and activates the CRC application. In automated mode, a computerized system of analysis determines that the threshold of criticality has been reached and automatically launches the CRC application. This computerized method of analysis can take a number of forms including (but not limited to) pre-defined rules or artificial intelligence techniques utilizing natural language processing. An alternative automated trigger for CRC activation can also be imposed by the referring clinician by requesting $\mathrm{CRC}$ status when placing the order for the imaging study. If an automated trigger opens up the $\mathrm{CRC}$ application, the radiologist has the ability to manually override and cancel the CRC application, but in doing so creates an electronic receipt of cancelation, which goes into the CRC database for subsequent review and analysis.

Once the application has been opened, the radiologist is tasked with the following input requirements:

1. Identification and annotation of a single or multiple key images.

2. Recording of mandatory supporting data (which is standardized).

3. Option to record additional non-standardized data.

4. Verification and/or editing of CRC recipients.

The selection of key images can be as simple as a single input command (e.g., speech command "save key image," right click on multi-programmable mouse, or selection of key image icon from UI toolbar). Once the key image/s is selected, the radiologist is tasked with annotating the image by marking the specific region of interest, along with the option for additional annotations (e.g., size, attenuation coefficient, morphology, etc.). The mandatory standardized data fields can be accessed by activating the "supporting data" field through a number of input options (e.g., speech command, mouse, icon, etc.). Once activated, the requisite data fields are presented for review, with or without the supporting options. The mandatory data fields and associated options are listed in Table 2. An ontology can be created to support the categories of finding/ diagnosis and anatomic location. In the near future, computerized anatomic localization (following annotation of the region of interest) would be a likely option. As stated in the companion article (1), the creation of finding specific macros could provide a fast and efficient means for providing these data, along with the ability to incorporate supplemental descriptive data (e.g., size, morphology, contrast enhancement, etc.). The ability to add non-standardized data can be performed in a supplemental data box, which accommodates typed or spoken text. In addition to descriptive supplemental data, another optional data field for linked data could be activated in which ancillary data in the patient's imaging or 
Table 2 Mandatory standardized data fields for CRC

\begin{tabular}{ll} 
A. Finding or diagnosis & \\
B. Anatomic location & \\
C. Classification & \\
1. & Emergent \\
2. & Discrepant \\
3. & Unexpected \\
4. & Clinical request \\
D. Urgency & \\
1. & Hyper-acute $(<1 \mathrm{~h})$ \\
2. & Acute $(<6 \mathrm{~h})$ \\
3. & Subacute $(<24 \mathrm{~h})$ \\
4. & Routine $(<72 \mathrm{~h})$ \\
E. Follow-up recommendations & \\
1. & \\
2. & Intervention \\
3. & Medical treatment \\
4. & Imaging exam \\
5. & Lab/clinical testing \\
\hline
\end{tabular}

${ }^{\text {a }}$ Once the follow-up recommendation category has been selected, the end user has the option for inputting additional descriptive information (e.g., surgical biopsy, chest $\mathrm{CT}$, and neurosurgical consultation)

clinical medical folders could be added by implementing a cut and paste option. The net result is an annotated image with accompanying standardized data defining the finding/ diagnosis, classification category, anatomic location, urgency, and follow-up recommendations.

Before closing the CRC application, the radiologist is presented with a computer-derived hierarchical list (i.e., rank order) of recipients. If accepted "as is," the computer will use this list to transmit the CRC and engage the escalation pathway in accordance with institutional guidelines commensurate with the urgency of the critical finding. Alternatively, the radiologist has the option to modify this recipient list by adding, removing, or adjusting the rank order of recipients (note that all modifications are recorded in the CRC database.). Once the recipient list has been finalized, the radiologist enters the "complete" command and the CRC transmission is begun. All actions are time stamped and recorded in the database, along with the identity of the responsible end user (using biometrics for authentication/identification). In addition to being recorded in the central CRC database, the CRC data is also recorded in duplicate in the patient imaging folder for direct access.

The transmission of CRC data is established by defined rules established by the institution, individual clinical care providers, and societal standards and incorporates an escalation pathway to ensure timely and reproducible CRC receipt. While each clinical care provider can establish his/her individual preferences as to the mode of CRC transmission; institutional, and societal standards (based upon best practice guidelines) will dictate $\mathrm{CRC}$ receipt, acknowledgement, and follow-up action time requirements. The timing of when the escalation pathway is automatically activated can be determined in several ways which include (but are not limited to) societal and/or institutional guidelines, historical records of the clinical care provider in question (provided by analysis of the CRC database), and defined time urgency and classification of the CRC in question. In addition to automated activation, the escalation pathways can also be manually activated by the primary clinical care provider in the event that they are not available for rapid CRC response. Examples may include the surgeon who is actively engaged in surgery, an ER physician who is taking part of an emergent resuscitation, or primary care physician who has recently signed off clinical responsibilities to his on call colleague. In addition to a "clinical" escalation pathway, which reacts to physician unavailability, a "technology" escalation pathway is also created, which monitors the communication system used and notifies technical support staff (e.g., chief information officer) in the event that technical problems arise, such as losing Internet access. In this event, the technical escalation pathway would be triggered which facilitates alternative transmission options which circumvent the technical problem encountered. The CRC sender has the option to transmit CRC data to more than one recipient at any time. This can be done one of two ways, either selecting from the escalation pathway list or manually inputting the name of the desired recipient. In addition to clinical care providers, CRC data can also be sent to patients and/or their legal guardians. While this may not be practical in hyper-acute emergencies, it may have relevance in non-emergent critical result cases where short-term follow-up is required to ensure interval stability or improvement. Along the same lines, simultaneous CRC transmission to administrative and/or quality assurance personnel can also be performed, which may be of value in clinical situations where the sender is concerned about timely and/or appropriate follow-up.

Receipt and acknowledgment is perhaps the single most critical step in the CRC process for without it timely and effective clinical action (in accordance with the critical imaging results) does not take place. In conventional practice, combined data transmission and receipt are customarily documented as an addendum in the radiology report. This typically consists of 1-2 free text sentences recording the date and time of the communication, the identity of the communicating parties, and the specific finding/s being communicated. While the data can be analyzed using natural language processing (NLP) for longitudinal analysis, most existing information systems and reporting technologies do not support creation of a standardized database which prospectively records, tracks, and analyze these data. An additional and important feature of the proposed innovation is to go one step beyond 
and utilize the derived real-time data analytics to create customizable interventions aimed at promoting best practice guidelines and improved clinical outcomes. One such intervention might consist of real-time modifications to the escalation pathway in accordance with the severity and context of the CRC. An example may consist of an acute ruptured appendicitis in a child with tachycardia. Based on the heightened emergency and criticality of timely surgical intervention, the system may respond by simultaneous emergent CRCs to the surgeon, anesthesiologist, and operating room nurse administrator on call. This would attempt to speed up clinical action and mobilization of the operating room staff for improving clinical outcomes in an extremely high risk clinical emergency. The proposed innovation serves to standardize the receipt and acknowledgment step in the cumulative CRC process, while also adding knowledge and understanding. In the current practice, it is not unusual for there to be a cognitive disconnect in the communication process, such that the party receiving the critical results data may not have a complete or accurate understanding of the data and/or clinical ramifications. This important component of "data understanding" is promoted in several features of the innovation which include the following:

1. Mandatory inclusion of standardized data reporting CRC classification, urgency, and follow-up recommendations.

2. Integration of a standardized system for documenting CRC understanding (Table 3).

3. Integration of a multi-directional consultation tool which provides multiple input options (e.g., image annotation, shared cursor, speech, and text) with the ability to capture consultation data.

In order to illustrate the standardized options for CRC data understanding, we will take a common example of a CT report with the finding of acute (low grade) appendicitis, which has been categorized as emergent (classification), acute (urgency), and requiring surgical intervention (follow-up recommendation). The surgeon receiving the CRC may determine that upon review, he/she fully understands the information provided and is in agreement with the diagnosis and recommendations. As a result, he/she responds with understanding option 1 (i.e., understanding complete, agrees with analysis and recommendations) and no further action is required by either party. In the event that the surgeon fully understands the

Table 3 Standardized method for documenting data understanding

1. Understanding complete, agree with analysis and recommendations

2. Understanding complete, disagree with analysis and recommendations (provide additional data)

3. Understanding complete, consultation requested

4. Understanding incomplete, consultation required
CRC data but disagrees with the diagnosis and/or follow-up recommendations, he/she may select understanding option 2 (i.e., understanding complete, disagree with analysis and recommendations [provide additional data]). In this example, the surgeon is aware that the patient has had a previous appendectomy and the diagnosis is therefore incorrect and provides this feedback to the interpreting radiologist. All data associated with the CRC is automatically recorded in the database (providing for longitudinal analysis), including the identity of the parties authoring and receiving data along with date/time stamps of all actions. In this example, the radiologist may react to the surgeon's feedback by modifying the radiology report with a new differential diagnosis (e.g., Crohn's disease, diverticulitis, and typhlitis) and corresponding changes related to classification, urgency, and follow-up recommendations. Alternative responses by the surgeon may consist of option 3 (i.e., understanding complete, consultation requested) or option 4 (i.e., understanding incomplete, consultation required). In one case (option 3),the surgeon has complete understanding of the CRC data but requests consultation with the radiologist (e.g., to collectively review the imaging data). In the other case (option 4), the surgeon does not fully understand the CRC data and this by definition requires additional consultation between the two parties. Once this consultation has been completed, the surgeon would in turn convert his/her CRC data understanding option from 4 to either option 1 or 2 in accordance with agreement between the two parties. Since the CRC database would record all responses and communications, the serial change in the surgeon's response would be recorded along with consultation data. This in effect creates a reproducible timeline, recapitulation of the CRC data, and interaction between the communicating parties in the event that a third party wishes to review the sequence of events which transpired in the course of a clinical event (e.g., adverse outcome).

The consultation feature is an integral component of the proposed innovation and serves as a mechanism to bridge the proverbial gap in knowledge, understanding, and agreement which commonly exists in conventional practice. In the "old days" of analog medical imaging practice, film images mandated that a physician physically travel to the radiology department to review images and in doing so would frequently consult with the radiologist. This created a face to face opportunity to educate, share information, and improve understanding, which in many respects has worsened in digital practice. The proposed CRC application seeks to mandate improved communication and understanding (which can be performed electronically if desired), while also creating a quantitative and qualitative method for improved accountability. In order to be effective and adopted in everyday practice, the consultation application must be workflow efficient, easy to use, adaptive to the individual needs and preferences of different end users, and intuitive. Since face to face (or even telephone conversations) may often be disruptive to workflow, the consultation 
tool is designed to be directly integrated into end user workflow in a manner that commensurates with individual preferences and the criticality of the CRC data. If for example the CRC is categorized as emergent, hyperactive, and requiring immediate intervention, the heightened criticality would mandate immediate consultation which supersedes all elective consultation preferences. In this case, the mandatory and time sensitive consultation would be expedited as the top workflow priority and the involved CRC parties (i.e., sender and recipient) would be required to complete the consultation before any other computer-based actions can be performed. If on the other hand the consultation is categorized as unexpected, subacute, and requiring follow-up imaging, the consultation would be required to be completed within a designated time frame (e.g., $12 \mathrm{~h}$ ), but existing workflow is not forcibly interrupted. The CRC parties can be presented with a list of consultation options at the time of $\mathrm{CRC}$ receipt acknowledgment, and the combined input will in turn serve as the driver for the timing and method of consultation. As an example, if the two parties agree on a specific time for the consultation to take place (which can be performed electronically by selecting the consultation scheduling feature of the application), the CRC consultation tool will automatically notify the parties of the scheduled consultation at pre-determined time intervals (e.g., 15 and 5 min prior to the scheduled time) with the options to continue as planned or reschedule. In the event the reschedule option is selected, the identity of the part requesting rescheduling will be recorded along with the time of the rescheduled consultation (this provides a data-driven analysis of determining consultation reliability and compliance.). Once both parties are in agreement, the consultation application is engaged and the CRC data is simultaneously presented to both parties along with a synchronized cursor and toolbar which allows for both parties to navigate the imaging data in tandem and review data inputs from either party. When speech is used in the consultation, the voice files are recorded and incorporated into the CRC consultation database. The consultation cannot be completed until both parties agree and an "understanding is complete" action has been recorded. This ensures that no party can subsequently claim there was a data misunderstanding or failed $\mathrm{CRC}$ communication.

Once CRC receipt and understanding has been documented, the next step in the CRC process is clinical action, which is directly tied to the CRC follow-up recommendations. This can take a number of different forms including (but not limited to) orders for a clinician consultation, laboratory or clinical test, pharmaceutical, imaging study, or clinical action (e.g., remove feeding tube). The common feature for any of these actions is the physician order, which takes place in information system technologies (e.g., most often in the electronic medical record, but potentially in other information systems such as the laboratory information system, radiology information system, pharmacy information system, etc.). The recommendations can be associated with a template that contains a pre- determined set of actions related to each recommendation. Alternatively, the CRC system can present a list of actions that can be manually selected. A combination of these would represent a standard template that could be edited by individual physicians who were initiating the CRC.

The next step is to translate the pre-defined protocol/ template or edited template or newly generated list for follow-up and automatically initiate the appropriate orders after recording the orders that were generated in the CRC database. Given a tight integration with an electronic medical record or other order entry system, these orders could be automatically generated with final electronic sign-off by the person initiating the $\mathrm{CRC}$ to ensure the orders were placed correctly required in most cases. Alternatively, the CRC system could generate a script or macro that would electronically sign into one or more order entry systems with the user's credentials and place each order pausing for electronic signature. These orders could be generated at the level of the user's portal or could potentially more directly interface with the system using HL7 or using a service oriented architecture or another communication protocol. Once the information system has been engaged, the corresponding order is automatically generated based upon the recorded (and agreed) CRC data, along with the patient's identification and clinical data (e.g., chest CT angiography to evaluate for pulmonary embolus). The entire automated ordering process could be triggered by having the end user simply activate the "order now" feature of the CRC application, which would in turn present the end user with the computer-generated order for modification and/ or completion of one or more orders. The selection function could in turn have biometrics integration for authentication and identification of the end user placing the order in lieu of a signature which could either be directly recognized by the order entry system or alternatively the biometric device could be used to identify the ordering physician which would then automatically generate what appears to the order entry system as a digital signature. All of this would be automatically recorded in the CRC database. This ordering function can at any time be converted from automated to manual operation, thereby allowing the end user to manually input the order in a more interactive and customary fashion such as the way in which orders are generally created by the order entry system(s). In some cases, automated ordering would allow additional end user input for order clarification when multiple options exist and cannot be readily determined by computerdriven artificial intelligence and predictive analytics. An example would consist of the appendicitis driven follow-up recommendation for surgical consultation. A number of different options may be presented for determination of a surgeon to select for a consultation for example and include (but not limited to) the following:

1. Manual input of name of surgeon for consultation. 
2. Computer-derived names of surgeons (in rank order) based upon historical usage of the ordering end user, clinical context, and surgeon availability.

3. Computer selection of "on call" surgeon.

4. Computer-derived name of surgeons (in rank order) based upon CRC performance analytics (e.g., responsiveness, timeliness, and clinical outcomes).

Once the resulting clinical action has taken place, it remains undetermined as to whether the reported critical result was indeed accurate. This necessitates an additional step in the collective CRC process, which is classified as diagnostic confirmation. In this step, the clinician/s acting upon the CRC will ultimately have the ability to confirm diagnostic accuracy based upon a number of potential data sources including (but not limited to) response to clinical treatment, surgical intervention, pathology results, medical surveillance and monitoring, and follow-up clinical and/or imaging data. This effectively serves as the final data point to measure clinical outcomes and aid in the creation and refinement of "best practice" guidelines and standards. The data recorded in this step of the CRC process would include the identity of the clinical care provider establishing diagnosis, the validity of the original CRC diagnosis, data sources used in diagnostic assessment, the date and time diagnosis is established, and ensuing clinical outcomes (i.e., response to treatment/ intervention). Since the latter data will largely be determined through analysis of unstructured data (e.g., hospital discharge summary and progress note), this will require additional technology such as NLP for data extraction and categorization. The core data component of this diagnostic confirmation step is found in Table 4, which provides four standardized options for the clinician to select from which provides clinical feedback as to whether the CRC radiologic diagnosis was confirmed clinically. These options include agreement, disagreement, or uncertainty and can be supported by supplemental data contained in the patient electronic medical record, which can be attached to the CRC application in a manner similar to e-mail attachments. When the "supplemental data" option is engaged by the clinician at the time of diagnostic confirmation

Table 4 Standardized method for assessment of CRC diagnosis and communication

$\begin{array}{ll}\text { 1. } & \text { Agree with initial diagnosis, without qualifications } \\ \text { 2. } & \text { Agree with initial diagnosis, with qualifications } \\ \text { 3. } & \text { Disagree with initial diagnosis } \\ \text { a. } & \text { Uncertain diagnosis }^{\mathrm{a}} \\ 5 . & \text { Failure to communicate critical result } \\ \text { a. } & \text { Missed critical finding } \\ \text { b. } & \text { Failure to communicate critical report data }\end{array}$

${ }^{\text {a }}$ Additional data required which can consist of unstructured free text and/ or supplemental report data from the EMR assessment, the end user is provided with the ability to select options from a pick list which is created in direct context to the reported CRC finding/diagnosis using computer artificial intelligence (e.g., neural networks), predictive analytics based upon historical use, or pre-defined rules. Alternatively, the end user can directly input the supplemental data source of interest through speech or written text and the computer will retrieve the corresponding data field for manual selection. Data analytics derived from this step provide valuable clinical diagnostic accuracy feedback to the reporting radiologist which is largely lacking in conventional practice and serves as a potential source of repetitive error. While not directly applicable to prospective CRCs, a fifth data point is captured in this database which refers to radiology report data which should have generated a CRC but did not. This could consist of either a "missed" critical finding or one that was included in the report but did not trigger an expected CRC. The method for reporting these "missed CRCs" could consist of a report application (i.e., missed CRC) which allows for the end user to provide direct feedback to the CRC database in either case. Due to the fact that this has important clinical implications, the recording of a missed CRC would automatically trigger an alert to the reporting radiologist, radiology department chief, and/or medical chief of staff. A mandated review would be required to validate or invalidate the reported missed CRC and if indeed found to be accurate and requires immediate clinical action.

The final step in the CRC process is data analysis (Table 5), which is a direct product of the standardized CRC database created by the proposed innovation. The primary metrics include accountability (i.e., responsiveness), timeliness, accuracy, compliance (with established standards, guidelines, and institutional policies), follow-up, and clinical management. These metrics can be analyzed on individual, departmental, and institutional levels and used to determine relative
Table 5 Components of CRC data analysis 
strengths and deficiencies within the $\mathrm{CRC}$ process, along with opportunities for improvement. When deficiencies are identified and interventional strategies are employed, the dynamic nature of the database provides a means with which objective real-time data can be used for temporal trending and rapid determination as to whether the interventional strategy employed has resulted in the desired outcome and improvement. Due to the fact that the collective CRC process consists of multiple steps and players, the database and derived analytics provide a mechanism for accounting for confounding variables and interaction effects. This provides an objective tool in which individual data outliers can be identified and parsed from the other data variables, so as not to draw negative conclusions on the entire CRC process. An example might consist of the operating room in a hospital which is slow in mobilizing and responding to acute surgical emergencies. If the surgeon responds to the CRC information promptly and accurately, it would be unfair to penalize the individual for the deficiencies of the operating room administrative and support staff. Another frequently encountered example is that of institutional deficiencies related to "after hours" staff and technology availability. In the examples of a head CT with questionable stroke or spine CT with questionable spinal cord compression, the follow-up recommendation often consists of an MRI, which may not be readily available until several hours later. This forces the referring clinician to either wait until local resources are available or transfer the patient to another facility, which causes additional time delays and cost, along with fragmentation of care. The ability to record and analyze institution-specific data within the CRC database and comparatively analyze relative to national norms (within the respective peer group) provides a valuable tool for resource allocation, technology procurement, and refinement of practice standards and guidelines.

\section{Designing an Implementation Strategy}

While the CRC innovation proposal attempts to create a comprehensive multi-step solution with the creation of a standardized CRC database, the practical solution may begin in a more finite and targeted approach. Any one of the individual steps in the CRC process could be used for targeted innovation, which could provide an opportunity for incremental quality improvement. As an example, a PACS vendor may create an application which provides radiologists with a one- step tool for saving key images, along with a standardized annotation schema for marking up these key images for integration into the radiology report. An EMR vendor may provide an electronic tracking tool which allows end users to document transmission and receipt of critical results communications, which in turn is automatically downloaded into a CRC database. A radiology information system vendor may integrate biometrics so as to unequivocally authenticate and identify each individual end user at the time critical results data is accessed and record the data being reviewed. The point is that creating an "all inclusive" solution may not be a realistic expectation at the present time, but a more reasonable one may consist of an incremental approach of creating individual components, which can in turn drive development of additional synergistic applications, which can eventually be coalesced into a single comprehensive solution.

The important innovation drivers to keep in mind are timing, economics, and burgeoning mandates. As greater emphasis is placed on data-driven quality and safety in medical practice as a service differentiator, economic incentives will promote innovative technologies which can objectively document improvements in timeliness, cost efficiency, and clinical outcomes. Critical results provide a unique opportunity to dramatically impact healthcare outcomes due to the urgency and magnitude of the stakes at hand.

\section{References}

1. Reiner B. Innovation opportunities in critical results communication: practical solutions. J Digit Imaging 2013 (in press)

2. Reiner B. Innovation strategies for radiology reporting and communication. Part 2: Using visual imagery for enhanced and standardized communication. J Digit Imaging 2013 (in press)

3. Reiner B: Customizing medical data. J Digit Imaging 23:363-373, 2010

4. Parl FF, O'Leary MF, Kaiser AB, et al: Implementation of a closedloop reporting system for critical values and clinical communication in compliance with goals of the Joint Commission. Clin Chem 56:417423, 2010

5. Ti LK, Ang SBL, Saw S, et al: Innovative strategy for effective laboratory result management: end-to-end process using automation and manual call center. BMJ Qual Saf 21:657-662, 2012

6. Singh H, Sethi S, Raber M, et al: Errors in cancer diagnosis: current understanding and future directions. J Clin Oncol 25:5009-5018, 2007

7. Arora V, Johnson J, Lovinger D, et al: Communication failures in patient sign-out and suggestion for improvement: a critical incident analysis. Qual Saf Health Care 14:401-407, 2005 